\title{
Sound wave and laser excitation for acousto-optical landmine detection
}

\author{
J.C. van den Heuvel $^{\mathrm{a}}$,V.Klein ${ }^{\mathrm{b}}$, P. Lutzmann ${ }^{\mathrm{c}}$, F.J.M. van Putten ${ }^{\mathrm{a}}$,M. Hebel ${ }^{\mathrm{c}}$, H.M.A. Schleijpen ${ }^{\mathrm{a}}$ \\ ${ }^{\mathrm{a}}$ TNO Physics and Electronics Laboratory, Oude Waalsdorperweg 63, 2597 AK The Hague, The \\ Netherlands \\ ${ }^{\mathrm{b}}$ Kayser-Threde GmbH, Wolfratshauser Str. 48, 81389 Munich, Germany \\ ${ }^{c}$ Forschungsinstitut für Optronik und Mustererkennung (FGAN-FOM), Gutleuthausstrasse 1, 76275 \\ Ettlingen, Germany
}

\begin{abstract}
Acoustic landmine detection (ALD) is a technique for the detection of buried landmines including non-metal mines. An important issue in ALD is the acoustic excitation of the soil. Laser excitation is promising for complete standoff detection using lasers for excitation and monitoring. Acoustic excitation is a more common technique that gives good results but requires an acoustic source close to the measured area. In a field test in 2002 both techniques were compared side by side. A number of buried landmines were measured using both types of excitation. Various types of landmines were used, both anti-tank and anti-personnel, which were buried at various depths in different soil types with varying humidity. Two Laser Doppler Vibrometer (LDV) systems of two different wavelengths for the different approaches were used, one based on a He-Ne laser at $0.633 \mu \mathrm{m}$ with acoustic excitation and one on an erbium fiber laser at $1.54 \mu \mathrm{m}$ in the case of laser excitation. The acoustic excitation gives a good contrast between the buried mine and the surrounding soil at certain frequencies. Laser excitation gives a pulse response that is more difficult to interpret but is potentially a faster technique. In both cases buried mines could be detected.
\end{abstract}

Keywords: landmine detection, buried landmine, non-metal mine, laser Doppler vibrometer

\section{INTRODUCTION}

Reliable and rapid detection of buried land mines - be it antitank mines (ATM) or antipersonnel mines (APM) - was and is a challenging task for the military as well as the civilian community. In this paper a comparison is made of two acoustic detection techniques: acoustic excitation and laser excitation with a Laser Doppler Vibrometer (LDV) for the detection of surface vibration. Both techniques are valuable in a sensor fusion concept since they provide complementary information about buried objects with respect to GPR or metal detectors.

The acoustic excitation or SLDV (scanning LDV) technique is a promising detection method to contribute to the challenging task of buried land mine detection. However, it has to be stressed that its main use is as an additional verification sensor in a sensor fusion concept. Metal mines as well as plastic mines (e.g. anti-personnel) can be detected, since this approach is absolutely independent from any metal content within a mine. This is valid for a variety of soil types including soils with ferromagnetic content. With ground-penetrating radar, objects embedded in the soil present a dielectric variation and cause a reflection of the electromagnetic wave. This permits a 3D-mapping of the ground, but does not give specific frequency "fingerprints" from objects as does the 2D-mapping by the SLDV with acoustic excitation. The acoustic excitation gives a good contrast between the buried mine and the surrounding soil at certain frequencies. The second technique of laser excitation gives a pulse response that is more difficult to interpret than the acoustic excitation but is potentially a faster technique and besides the shape and size information additional depth information could be detected.

In this paper results of a combined field test of September 2002 are presented. The relative merits of the two techniques are discussed and their potential for use in land mine detection system are assessed. 


\section{TEST SITE}

The experiments have been performed in September 2002 at the test facility for landmine detection systems situated near TNO-FEL. ${ }^{1}$ The test facility consists of outdoor test lanes and a sand area that is shielded by a large tent. During these measurements the weather was quite stable without rain and sunny periods.

\subsection{Description of the test lanes}

The facility consists of six test lanes of 30 square meters each $(10 \times 3 \times 1.5 \mathrm{~m})$. Four lanes are filled with a native soil with original structure and texture. These soils include sand, clay, peat and a ferromagnetic soil. A fifth lane is filled with a sandy soil with forest remnants like roots and twigs. A sixth lane is filled with a sandy soil but also has a vegetation cover. The soils of the other lanes are bare.

Each type of soil has been characterized. A chemical and physical description is available which gives insight in the texture and structure of the different soil types. The test lanes are completely constructed without the use of electrically conducting material. A zone of 5 meters around the test lanes is cleared from all metal.

A set of test objects, representing anti-personnel mines, anti-tank mines, and false targets (stones, cans, etc.) have been placed at various depths $(0-30 \mathrm{~cm})$ in the test lanes. The mines are made of various materials and have different shapes and sizes. Non-metal mines are included.

The test mines have signatures close to those of real mines. To simulate the explosives, the devices have been filled with a silicone rubber. Experiments have proven that this is an excellent surrogate of TNT since both substances have the same electromagnetic and thermal characteristics. In our experiments the vibration issues of the mine surrogates are of crucial importance. Therefore, original mine casings were used for the surrogate mines.

\subsection{Description of the shielded sand area}

A large tent shields the sand area from rain and wind. It is used for short and flexible tests on landmines in contrast with the test lanes that have mines that have been buried for several years. Mines of identical type as buried in the test lanes were buried in the shielded sand area. Figure 1 shows the mines that were used in the sand area.
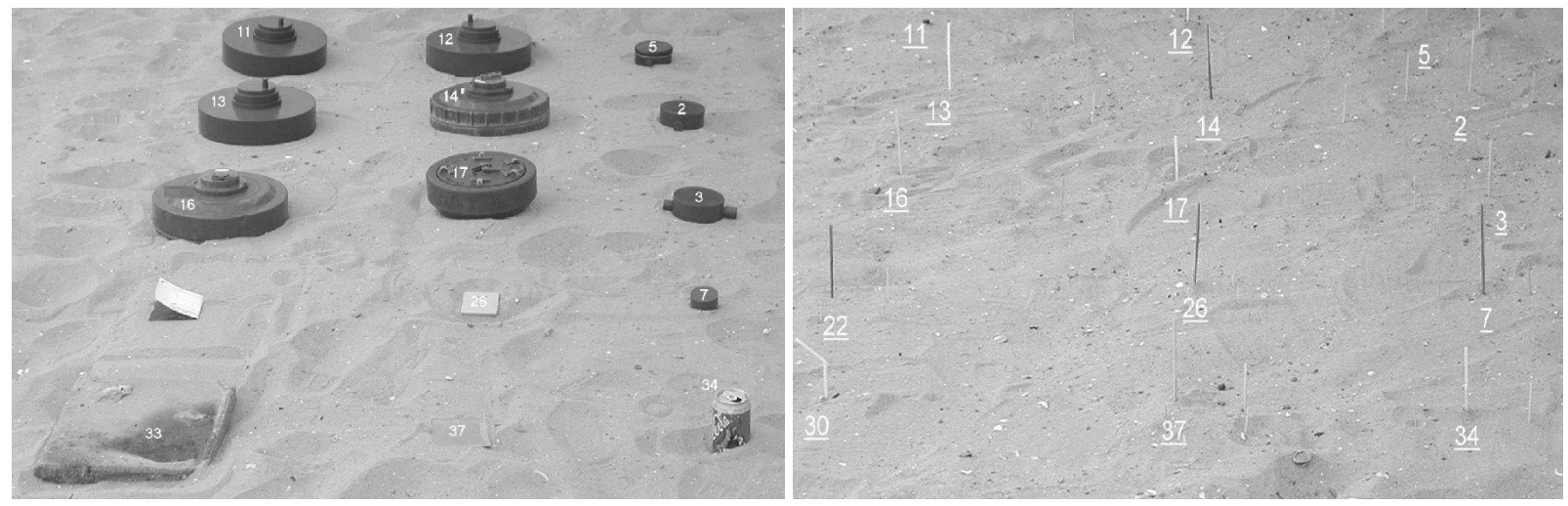

Figure 1: Mines before and after being buried in the sand.

\section{ACOUSTIC EXCITATION}

\subsection{Experimental set-up}

With the Scanning Laser Doppler Vibrometer (SLDV) technique, an acoustic source - e.g. a loudspeaker - is emitting its acoustic energy towards the ground (Figure 2). The SLDV instrument is investigating the surface of the ground from a typical distance of some $100 \mathrm{~cm}$ (may vary due to operational constraints). The sound source, mostly directly placed on the ground under a slight angle to it, excites slow speed vibrational Biot waves propagating downwards into the soil. These waves are characterized by strong attenuation (typical penetration depth $20-30 \mathrm{~cm}$ ) and high dispersion. In case a mine or other inhomogeneity is present below the soil surface the transmitted sound waves are scattered or reflected by 
the buried object. For targets very close to the surface, the scattered field is even indicating shape and size of the buried object. These surface vibration images are read out by the SLDV sensor, scanning the ground in a pre-programmed pattern. Upon completion of a sounding, the SLDV instrument is providing a data set whose structure can be described as 3-dimensional. This data set is depicted in the right part of Figure 2. Two-dimensional position data is combined with a third frequency dimension to provide a $3 \mathrm{D}$ data set.

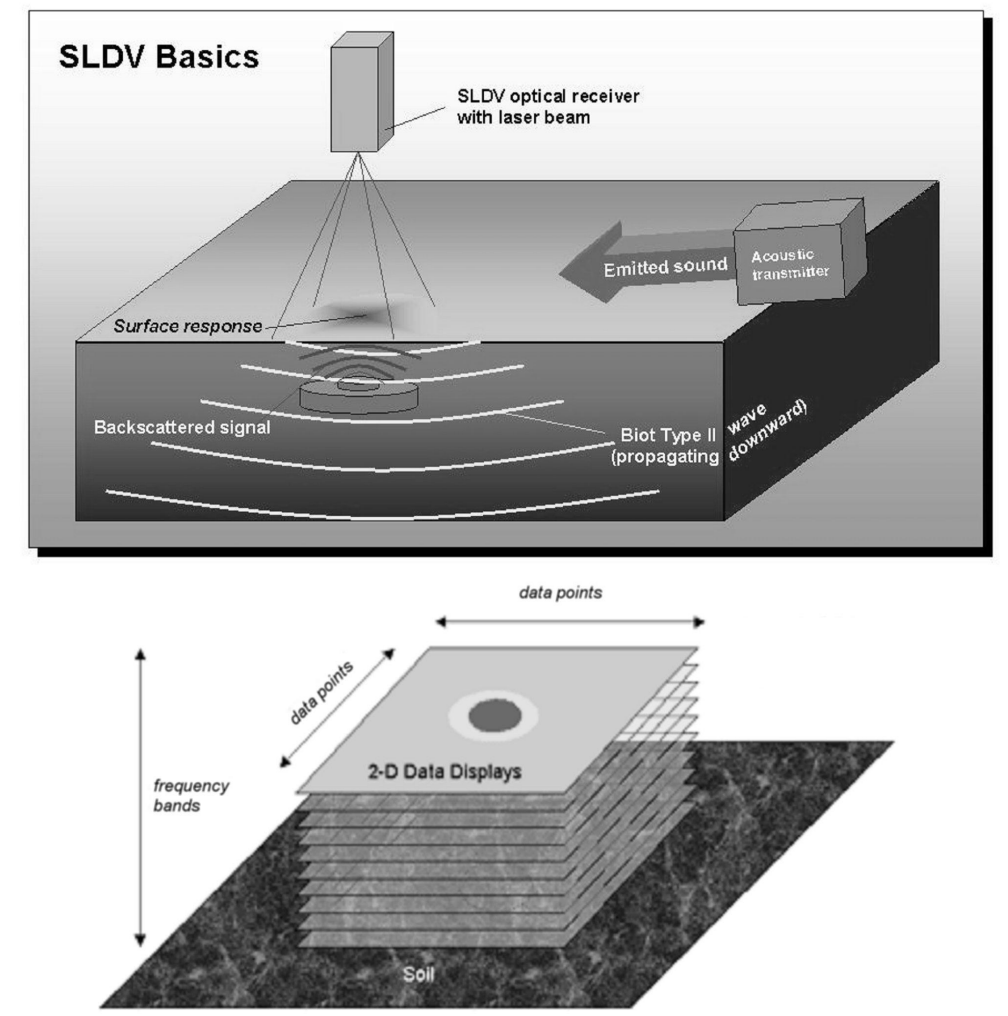

Figure 2: SLDV basics and 3D structure of SLDV data

Figure 3 shows the experimental setup of the acoustic excitation at the outdoor test lanes. The SLDV is a commercial device from the company of POLYTEC with a He-Ne laser source $(632 \mathrm{~nm})$. The key issue was the strong shielding for minimizing the impact of the external vibrations on the SLDV. A hi-fi loudspeaker was used for generating the sound (at about $105 \mathrm{~dB}(0 \mathrm{~dB}=20 \mu \mathrm{Pa})$ sound pressure level). The composition of this sound spectrum is determined by the spectral data that will be used during the sounding. All frequencies related to the different chosen frequency bands are feeded simultaneously in time (frequency comb) into the loudspeaker. Typical values are covering the spectrum from $40 \mathrm{~Hz}$ through $1 \mathrm{kHz}$. 


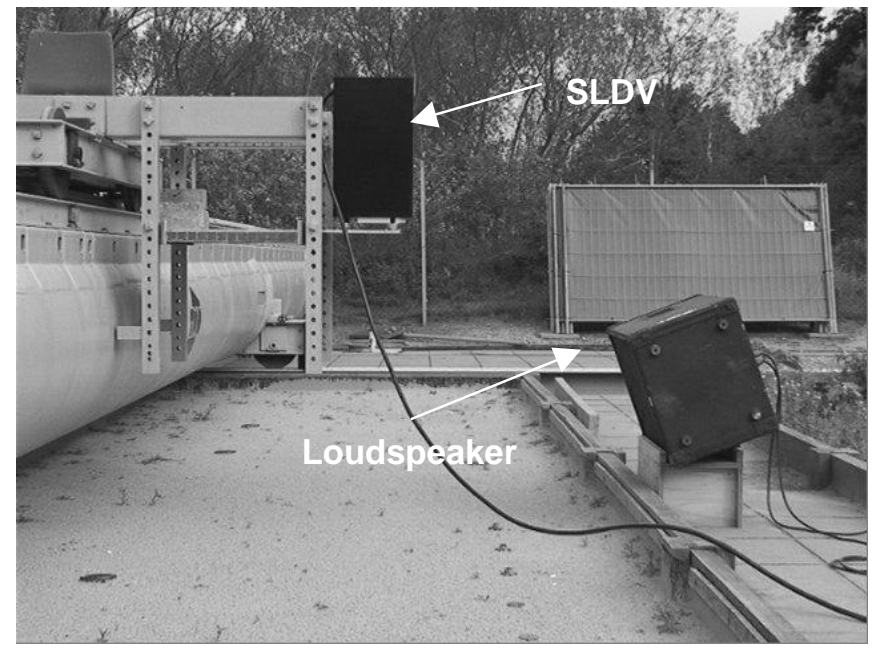

Figure 3: Experimental setup of the acoustic excitation at outdoor test lanes.

\subsection{Results}

The recorded vibration images and frequency spectra are characteristic for the type of mine (and different for other buried objects such as stones). The SLDV technique is detecting metal mines as well as plastic mines (e.g. antipersonnel), since it is absolutely independent from any metal content within a mine.

In several successful field trials, different mines and other objects were investigated under the influence of different types of soils (river gravel, loam, clay, sand or grass). During these field trials, situations with wet or moist soil conditions were encountered, situations that are less favourable for SLDV.

One current drawback is the measurement time needed. A typical multispectral scan of $1 \mathrm{~m}^{2}$ takes about 8 minutes (38x28 number of points), depending on the selected spatial resolution; but improvements are already being investigated using detector arrays or dedicated predefined acoustic frequencies.
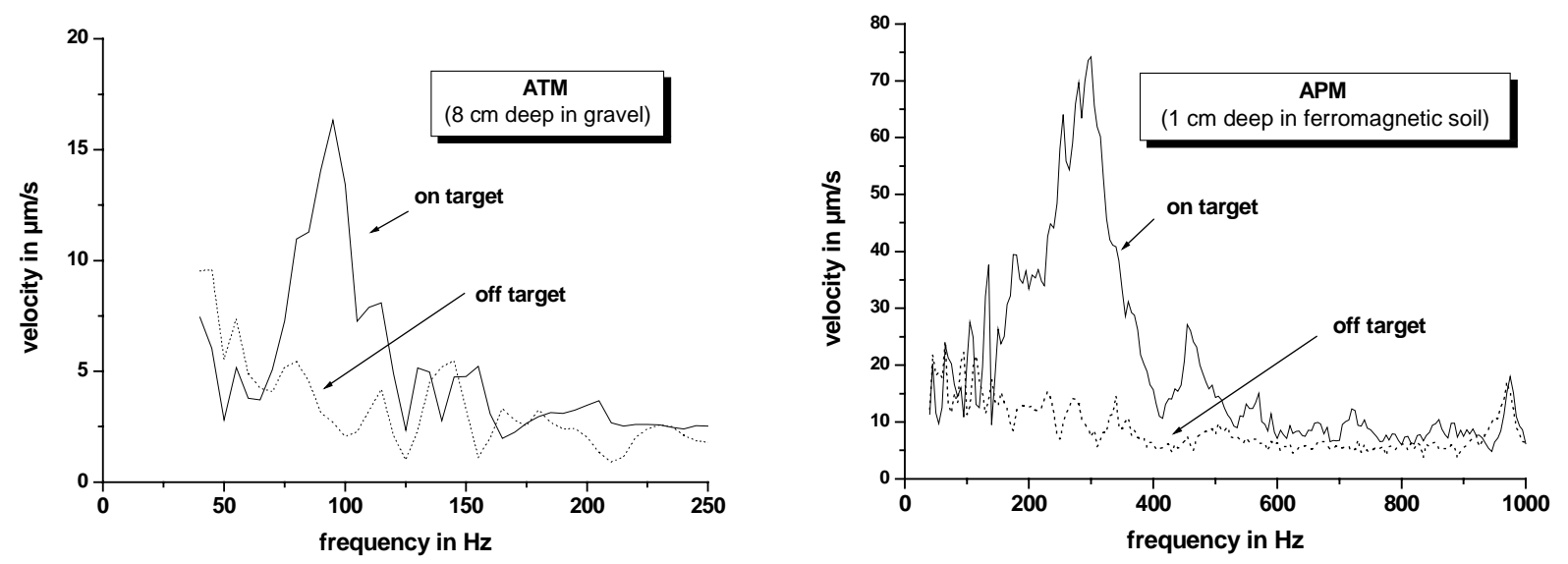

Figure 4: Spectral responses directly above the top of the ATM ( $8 \mathrm{~cm}$ deep in gravel) and the APM (1 cm deep in ferromagnetic soil) and besides the mines (off target / background). Note the different main frequency peaks of the two types of mines (ATM at $95 \mathrm{~Hz}$ and APM at $295 \mathrm{~Hz}$ ). Spectral resolution: $5 \mathrm{~Hz}$. The measurements were done with the He-Ne SLDV. 
Figure 4 reveals an illustrative plot of the velocity in frequency domain of two sampled locations (above the top of the mines and besides the objects). This shows the capability of such a SLDV system. SLDV soundings are clearly indicating an inverse correlation between size and spectral surface responds; smaller objects are scattering higher frequencies, whereas larger objects tend to enhance lower frequencies. The heavier ATM shows a lower frequency response (main frequency peak at $95 \mathrm{~Hz}$ ) compared to the lighter APM (main frequency peak at 295 Hz); the reason for that is the mine size but also the internal composition.

A presentation of a set of 2-dimensional intensity coded maps, showing the vibration intensities of the individual measurement points in a dedicated intensity scale, of the same mines are given in Figure 5. Special smoothing and filtering can additionally be applied to enhance these visualisations. The maps seen here are presenting data of two frequency bands for the ATM as well for the APM, based on an identical geometrical scale. Besides the different frequency response, information about the size and shape of the buried object were available. Of course, the possible determination of the shape and size are reduced by less resolution of the grid (number of scanning points) and deeper buried objects. Additional, more theoretical work was recently been published by Ning Xiang, et al. ${ }^{6}$

ATM
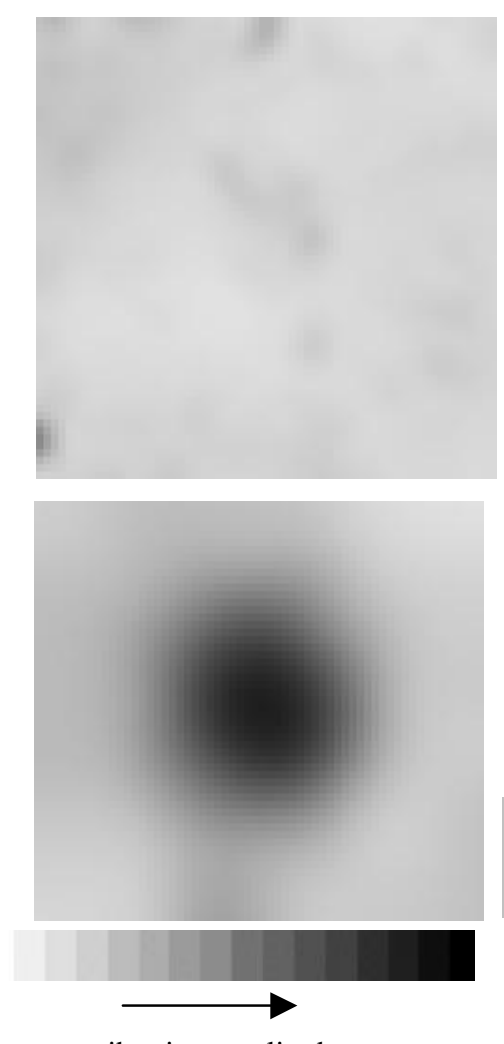

vibration amplitude
APM
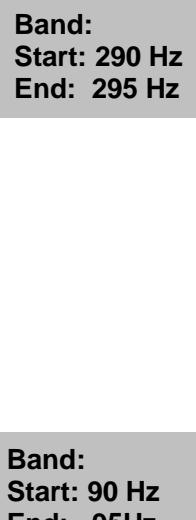

End: $95 \mathrm{~Hz}$

Figure 5: SLDV images for two different ranges of vibration frequency and two mine types (anti-tank and antipersonnel). ATM: $8 \mathrm{~cm}$ deep in gravel $\left(15 \times 14\right.$ points at a scan area of $\left.75 \times 65 \mathrm{~cm}^{2}\right)$ and APM, $1 \mathrm{~cm}$ deep in ferromagnetic soil $\left(31 \times 31\right.$ points at a scan area of $\left.50 \times 48 \mathrm{~cm}^{2}\right)$. Examining the same scenario at different frequency reveals the type of buried objects at specific frequencies. 

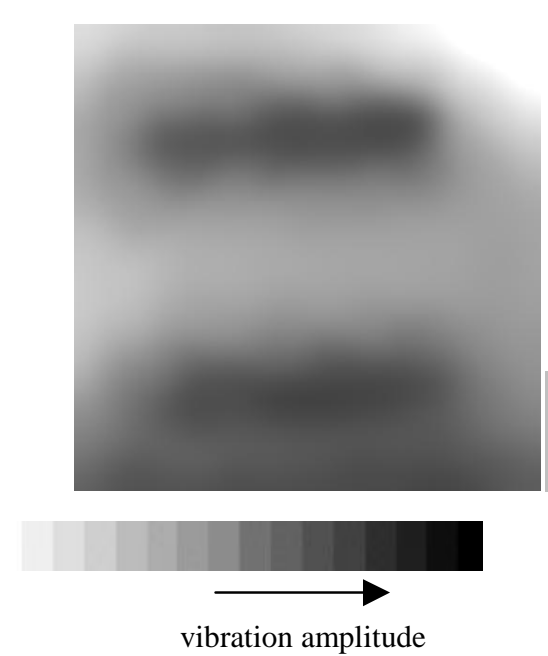
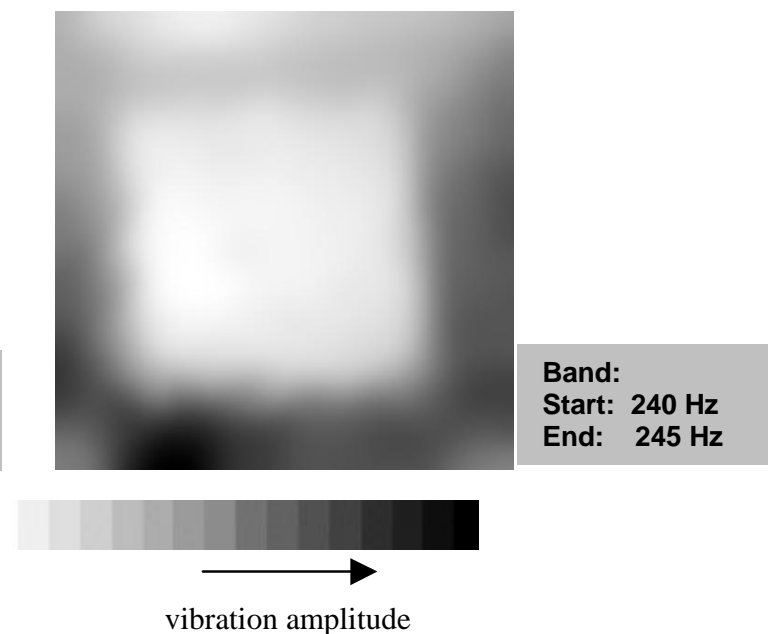

Figure 6: Concrete stone (pavement) buried in the sand test bed at $1 \mathrm{~cm}$ depth is showing a "negative contrast" at the higher frequency band (lower vibration excursions compared to the surrounding background). At the lower frequency band, only the two opposite edges are showing increased vibration intensities.

An another particular effect is shown by Figure 6. Not always an object shaped area of decreased vibration intensity is generated by the underground object. A thick concrete stone (pavement) was buried in the sand test bed at $1 \mathrm{~cm}$ depth. Lower vibration signals compared to the surrounding background occurred at certain frequencies. The plate can clearly been seen at the higher frequency band, but with "negative contrast"; whereas at the lower frequency band, only the two opposite edges are showing increased vibration intensities (the sounding by the loudspeaker was from the lower edge to the upper one).

\section{LASER EXCITATION}

\subsection{Experimental set-up}

Laser excitation is a promising technique for complete standoff detection using lasers for excitation and monitoring. With laser excitation a Q-switched laser pulse heats a small area of the surface of the soil in a very short time of a few nanoseconds. Due to the heating of the soil and the secondary heating of the air in and above the soil, an acoustic pulse is generated that propagates in the soil. The vibrations of the soil's surface are measured with an LDV at a range of several meters. These vibrations are modified due to the presence of a buried mine. ${ }^{3}$ The detection of the mine is based on the change in surface vibration. In first order the acoustic shock wave generated by the laser pulse reflects back from buried objects to the surface; the echo is measured by the LDV.

At present we do not use an integrated system but two separate laser systems: one for excitation and one for laser Doppler vibrometry. During research this is not a disadvantage, however, an operational system has to have an integrated excitation laser and monitoring laser. For the excitation of acoustic shock waves a Q-switched Nd:YAG laser was used. This laser (Quantel Brilliant) can deliver pulses with an energy up to $350 \mathrm{~mJ}$ with a $20 \mathrm{~Hz}$ rep-rate at $1.064 \mu \mathrm{m}$ (see Figure 7). A variable attenuator and focussing optics resulted in a laser spot of about $1 \times 3 \mathrm{~mm}$ on the sand with an energy of $170 \mathrm{~mJ}$. 


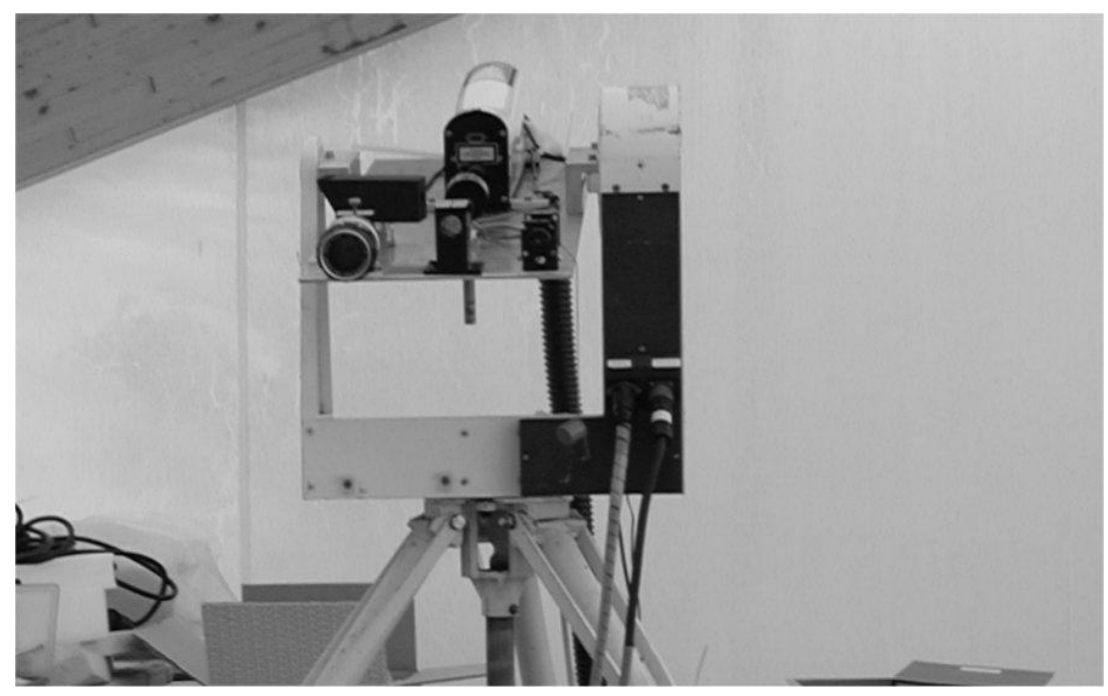

Figure 7: Nd:YAG laser for acoustic excitation.

The laser vibrometer used for the experiments was developed at TNO for long range vibration measurements with a 1 Watt $1542 \mathrm{~nm}$ laser source (see Figure 8). Because this high power is not necessary at the short range of a few meters, the power was reduced to about $175 \mathrm{~mW}$.
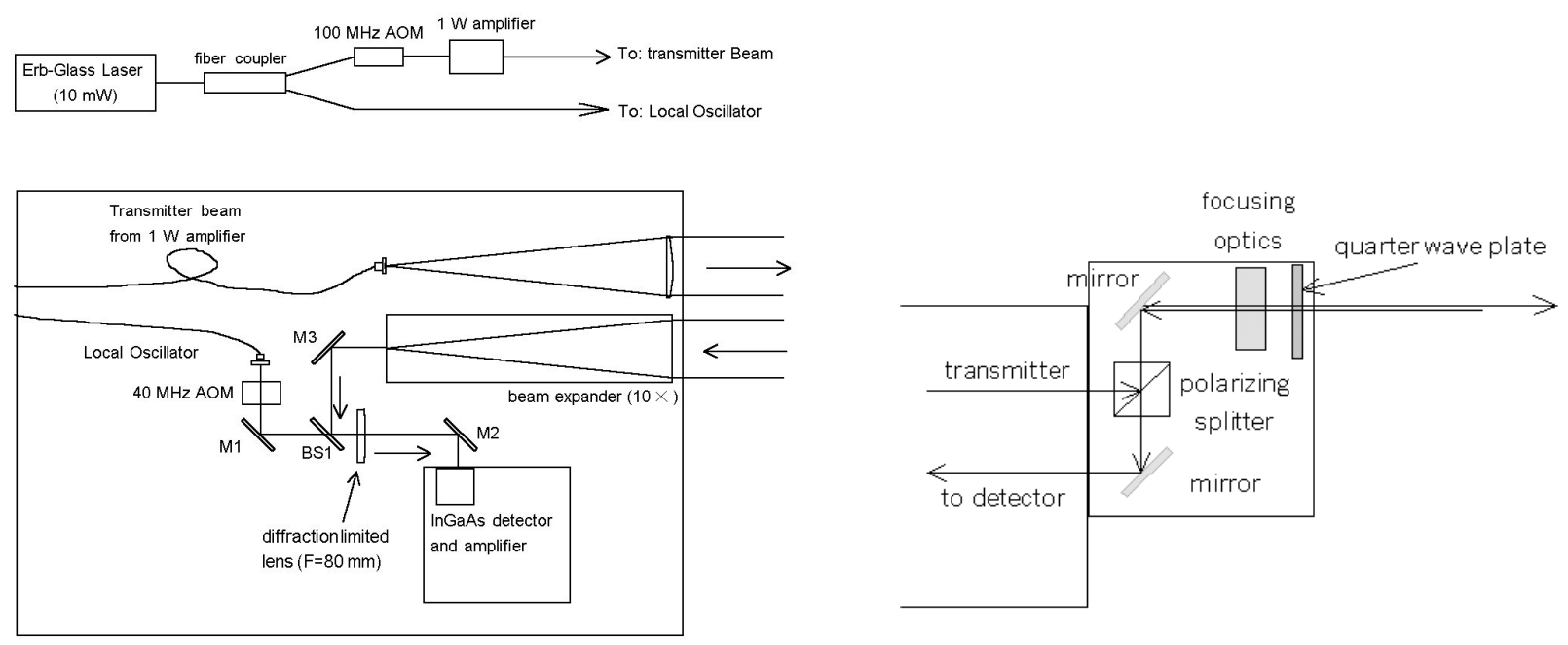

Figure 8: TNO eye-safe laser vibrometer with short range adaptation optics.

Due to the small divergence of the laser and the small field of view of the detector additional optics were placed in front of the system to measure at short distances. Using a polarizing beam-splitter cube and a quarter-wave plate monostatic operation was possible while 2 additional lenses were used to focus at short distances (see fig. Figure 8).

The vibrometer uses 2 Bragg cells (100 MHz and $40 \mathrm{MHz}$ ), resulting in a $60 \mathrm{MHz}$ frequency signal at the detector. This signal is mixed down to a frequency of $455 \mathrm{kHz}$. After amplification the signal is fed to a $10 \mathrm{Ms} / \mathrm{s}$ AD card and is stored on the computer for further analysis.

The relative position of the excitation laser spot and the monitoring laser spot has to be optimized. ${ }^{5}$ If the monitoring laser spot is too close to the excitation spot, the received signal is dominated by scattered soil particles that overwhelm the small response of the buried object. However, if the monitoring laser spot is too far away, the sensitivity for the 
acoustic signal is too low. In order to find the optimum position, the LDV was pointed at a number of spots around the spot of the $1.064 \mu \mathrm{m}$ laser as indicated in Figure 9.

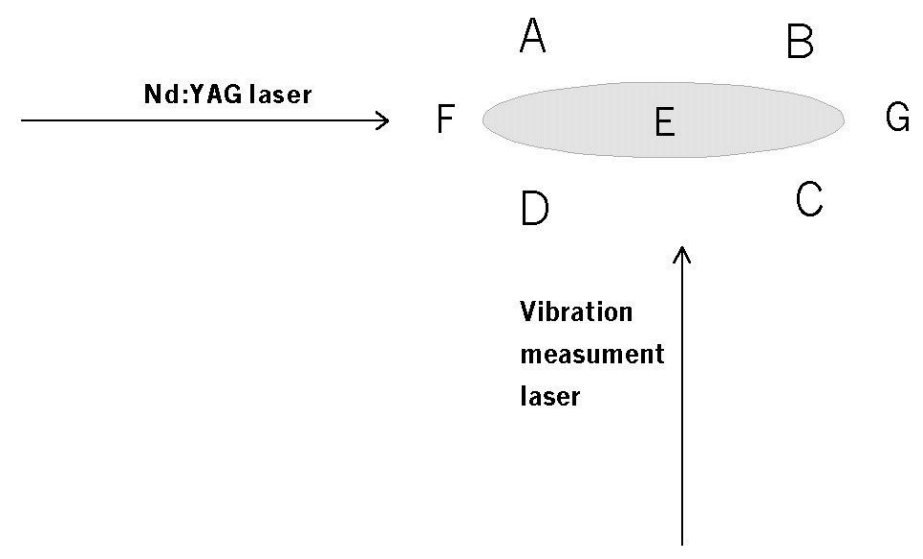

Figure 9: positioning of the laser on the test site

The LDV was aimed at points A to $\mathrm{G}$ at about $1 \mathrm{~cm}$ from Nd:YAG laser spot E. At every position vibration data from 16 consecutive pulses was collected by a computer.

\subsection{Results}

The acoustic signal results in a frequency modulation of the $455 \mathrm{kHz}$ high frequency carrier as a result of the Doppler frequency shift $2 v / \lambda$, where $v$ is the velocity and $\lambda$ is the wavelength of the laser. MATLAB was used to demodulate the high frequency signal in order to obtain the instantaneous velocity as a function of time.

The Nd:YAG laser was aimed at the center of mine 11 of which the center was $1 \mathrm{~cm}$ deep buried in the sand. Mine 11 is a test mine simulating the anti-tank mine TM62P. As a reference the Nd:YAG laser was also aimed at a position without a buried object. The demodulated signals are shown in Figure 10; the figures on the left and on the right are with and without mine, respectively. About $200 \mu \mathrm{s}$ after the data acquisition AD card is triggered the laser pulse hits the sand. A clear response is visible due to the surface excitation. Clearly, the LDV measures the acoustic wave created by the laser pulse (first peaks). After about $50 \mu$ s another signal is measured as the result of the reflection of the acoustic wave from the mine top. ${ }^{2}$ This delay corresponds quite well with the depth of $1 \mathrm{~cm}$ and an expected sound velocity of about $340 \mathrm{~m} / \mathrm{s}^{4}$ If there is no mine beneath the laser spots only the first signal peaks are measured by the LDV.
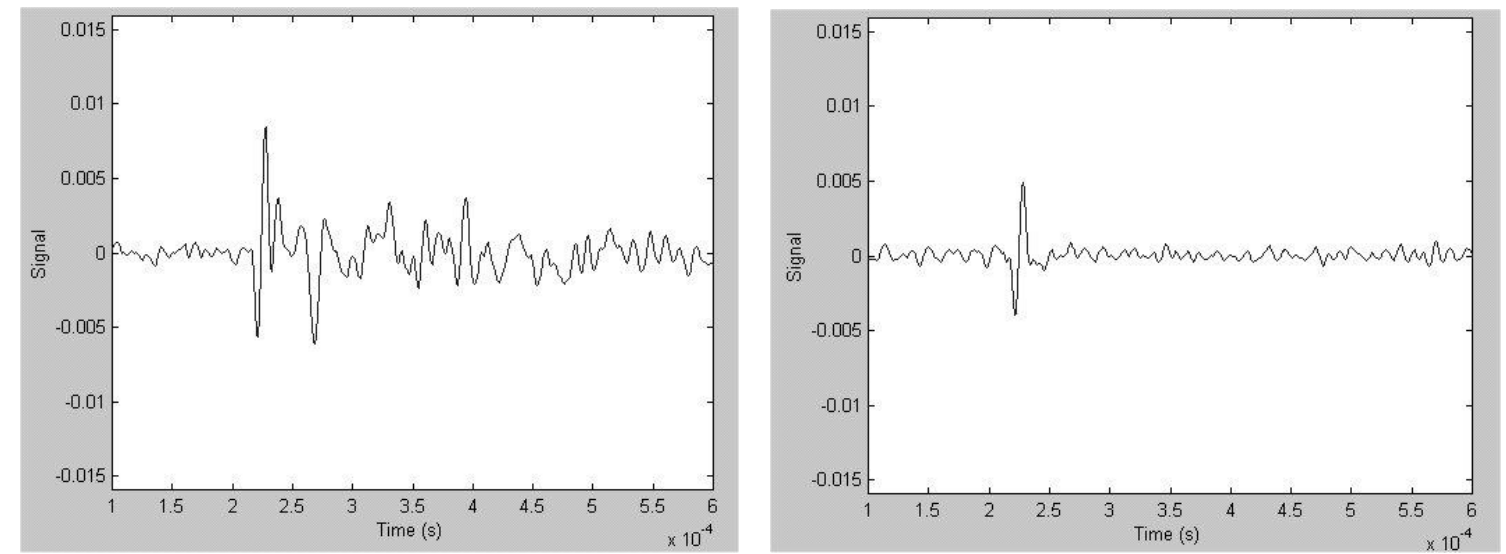

Figure 10: reflected vibration signal from mine 11 (TM62P) at $1 \mathrm{~cm}$ depth (left) and signal without mine (right). 
The response of the soil shows large variations from shot to shot, both in the first (surface) peak as in the reflection from the test mine. These variations make the interpretation of the data difficult. It is not clear yet whether it is possible to extract more from the data than the approximate depth of a buried object.

It was not possible to detect the presence of an object at a depth of more than $3 \mathrm{~cm}$. However our system has not yet been optimized and further research is required to find the limits of this technique.

\section{COMPARISON ACOUSTIC AND LASER EXCITATION}

The two detection techniques, acoustic excitation (AE) and laser excitation (LE), are not at the same level of technical maturity. The AE technique is much more mature than the LE technique. This is reflected in the almost operational system for AE during the field test and the modified laboratory equipment for the LE.

Both techniques do not depend on metal content for detection and are able to detect buried mines as was shown in the paper. These properties make them valuable for detection of buried land mines. In principle both techniques should be able to detect shape and size of the buried object by scanning the surface. However, in the case of LE this has proven difficult due to the shot-to-shot variations in the acoustic response of the soil. Further analysis of the data is required to assess whether it is possible obtain the shape of the object.

In the 'third' dimension both techniques differ, AE gives the frequency response of the buried object while LE gives the time response. For a first interpretation, the time response gives the depth of the mine, which is convenient for fusion with other sensors that provide depth information. The AE gives the frequency response, which could be used for classification of the buried object (mine). It is conceivable that the time response of the LE could also be used for further classification. However, this has to be shown in a more detailed analysis of the data.

Detection of deep objects seems restricted to AE. The typical results of this paper: $8 \mathrm{~cm}$ for AE and $1 \mathrm{~cm}$ for LE give a good indication of the difference. It is not expected that LE can detect objects deeper than $3 \mathrm{~cm}$. The excitation energy for LE is much lower compared to AE. In addition, the energy with LE is initiated at a small spot and will disperse over a larger area resulting in a low acoustic response.

Compared to the acoustic excitation, laser excitation is a fast technique. In principle, laser excitation gives a result within a few hundred microseconds, based on the depth of the buried object and the speed of sound in the soil. An operational system could be based on a high rep-rate excitation laser that gives a short pulse every 200 microseconds. With a shot spacing of $1 \mathrm{~cm}$, an area of $100 \times 50 \mathrm{~cm}$ could be covered in second. For a vehicle mounted system, this results in an operational speed of $0.5 \mathrm{~m} / \mathrm{s}$ with a swath width of 1 meter. As has been reported, AE required 8 min for a $1 \mathrm{~m}^{2}$ area. Thus, LE is, in potential, about two orders of magnitude faster than AE.

\section{CONCLUSIONS}

In September 2002 a field test of acoustic land mine detection was performed. Two acousto-optical techniques for the detection of buried landmines have been compared: acoustic excitation and laser excitation of the soil, both with a laser Doppler vibration (LDV) sensor. It has been shown that the techniques are complementary: acoustic excitation is a relatively slow technique that is excellent as a verification sensor, while laser excitation is a fast technique that is much less suited for identifying buried objects. Both techniques have shown promising results in the detection (and classification) of buried mines.

A more detailed analysis of the results of the field test is expected to provide more information on the relative merits of the two techniques. In this subsequent analysis we also plan to use the recorded data of buried microphones. These microphones were used to register the propagation of sound in the soil and provide information on the extinction and the dispersion of the acoustic energy.

\section{REFERENCES}

1. W. de Jong, H. A. Lensen, and Y. H. L. Janssen, "Sophisticated test facility to detect land mines," in Proc. SPIE Vol. 3710, Detection and Remediation Technologies for Mines and Minelike Targets IV, A. C. Dubey and J. F. Harvey, eds., pp. 1409-1419, (Orlando (FL), USA), Apr. 1999. 
2. W. Li, C.A. DiMarzio and S.W. McKnight, "Laser-induced Acoustic Detection of Shallow-Buried Objects", Proc. SPIE Vol.3752, July 1999.

3. S.W. McKnight et.al., "Laser-induced acoustic generation for buried object detection", Proc. SPIE Vol. 4038 (April 2000).

4. S.W. McKnight, J. Stott, C.A. DiMarzio, R Cleveland, R. Roy, "Laser-induced acoustic imaging of buried land mines: experiment and modeling", SPIE vol 4394, pp. 627-633.

5. Charles A. DiMarzio, et. al., Toward a Laser-Based, "Non-Contact Acoustic Landmine Imager"; Proc. SPIE Vol. 4038 (April 2000)

6. Ning Xiang, "Bayesian Probability Analysis for Acousto-Seismic Landmine Detection", Center for Physical Acoustics, University of Mississippi, Fall meeting of Acoustical Society of America (ASA), Cancun, December 2002 